\title{
DIRECT DETECTION OF THE BROWN DWARF GJ 802B WITH ADAPTIVE OPTICS MASKING INTERFEROMETRY
}

\author{
James P. Lloyd,${ }^{1}$ Frantz Martinache, ${ }^{1}$ Michael J. Ireland,${ }^{2}$ John D. Monnier, ${ }^{3}$ \\ Steven H. Pravdo, ${ }^{4}$ Stuart B. Shaklan, ${ }^{4}$ and Peter G. Tuthill ${ }^{5}$ \\ Received 2006 July 17; accepted 2006 August 31; published 2006 October 10
}

\begin{abstract}
We have used the Palomar 200" adaptive optics (AO) system to directly detect the astrometric brown dwarf GJ 802B reported by Pravdo et al. This observation is achieved with a novel combination of aperture masking interferometry and AO. The dynamical masses are $0.175 \pm 0.021$ and $0.064 \pm 0.032 M_{\odot}$ for the primary and secondary, respectively. The inferred absolute $H$-band magnitude of GJ 802B is $M_{H}=12.8$ resulting in a modeldependent $T_{\text {eff }}$ of $1850 \pm 50 \mathrm{~K}$ and mass range of $0.057-0.074 M_{\odot}$.

Subject headings: instrumentation: adaptive optics — stars: low-mass, brown dwarfs — techniques: interferometric

Online material: color figures
\end{abstract}

\section{INTRODUCTION}

Binary stars provide us with a unique laboratory for the study of the physical properties of individual objects and important constraints on star formation and evolution. Although there are now a large number of objects known beyond the substellar limit, there are few in systems amenable to extraction of dynamical measurements of physical parameters (Burgasser et al. 2006). This is a particularly acute issue in the case of substellar objects due to the degeneracy in age, mass, and luminosity.

We have selected targets from the Stellar Planet Survey (STEPS; Pravdo \& Shaklan 1996) that show astrometric evidence of a low-mass companion for imaging follow-up with adaptive optics (AO). In order to improve the sensitivity to companions, we have implemented a novel aperture masking interferometry technique in concert with the atmospheric turbulence correction afforded by AO. This technique has succeeded in directly detecting the low-mass companion to the M5.5 dwarf GJ 802.

\section{OBSERVATIONS}

\subsection{Aperture Masking Interferometry}

While the large gains of AO for high-contrast imaging are widely recognized (e.g., Macintosh et al. 2003; Carson et al. 2005; Close et al. 2005), nearly all AO searches for substellar companions have focussed on achieving very high dynamic range $\left(>10^{4}\right)$ at moderate separations. Several issues drive AO imaging to this parameter space. The interaction of the actuator count of AO systems and the limitations of coronagraphy (Sivaramakrishnan et al. 2001; Lloyd et al. 2001) lead to both practical and fundamental limitations for high-contrast imaging. Present and currently planned (Macintosh et al. 2004) AO systems are focused on achieving high contrast at radii of more than $4 \lambda / D$. At closer separations, diffracted light is difficult to suppress with a coronagraph, and most importantly there is a large noise floor due to the presence of fluctuating speckles in

\footnotetext{
${ }^{1}$ Department of Astronomy, Cornell University, Ithaca, NY 14853.

${ }^{2}$ Division of Geological and Planetary Sciences, California Institute of Technology, Pasadena, CA 91125.

${ }^{3}$ University of Michigan, Astronomy Department, 941 Dennison Building, Ann Arbor, MI.

${ }^{4}$ Jet Propulsion Laboratory, California Institute of Technology, 4800 Oak Grove Drive, Pasadeana, CA 91109.

${ }^{5}$ School of Physics, University of Sydney, Sydney NSW 2006, Australia.
}

the image (Racine et al. 1999; Fitzgerald \& Graham 2006; Soummer et al. 2006). Finally, it has proven to be remarkably difficult in practice to precisely calibrate the AO point-spread function (PSF). A variety of differential imaging approaches have been proposed to circumvent the problem of AO PSF calibration (Marois et al. 2000, 2004, 2006; Brandner et al. 2005; Sparks \& Ford 2002). These approaches rely on exploiting a differential signal in wavelength, polarization, or sky rotation to improve the source extraction but do not fundamentally address the issue of calibration of AO data.

In light of these considerations, we have undertaken a novel experiment to achieve precision calibration of AO data, by marrying the sensitivity of $\mathrm{AO}$ observations with the precision calibration afforded by interferometry. The heritage of nonredundant masking interferometry (Tuthill et al. 2000; Readhead et al. 1988; Nakajima et al. 1989) can be combined with the wave front stabilization of adaptive optics (Tuthill et al. 2006). In practice, the optical implementation of this capability is relatively simple (see Fig. 1). We have used the Palomar $200^{\prime \prime}$ telescope with the adaptive optics (PALAO) system (Troy et al. 2000) and the near-infrared camera system PHARO (Palomar High Angular Resolution Observer; Hayward et al. 2001). PHARO was designed with coronagraphic capability in mind and thus incorporates a 10 position Lyot wheel in the collimated beam at the internal position of the reimaged telescope pupil. This wheel holds a variety of pupil stops to enable the interchange of Lyot stops with various undersizings (Oppenheimer et al. 2000). We have installed nonredundant masks in the spare openings of the PHARO Lyot wheel. The nine-hole mask used in this work is optimized for broadband, faint targets with $50 \mathrm{~cm}$ diameter subapertures and $4.15 \mathrm{~m}$ longest baseline. The nine-hole mask transmits approximately $15 \%$ of the total light incident on the telescope pupil.

The advantages of this approach are several-fold. By preserving nonredundancy, a given baseline in the pupil formed by any pair of subapertures translates uniquely to a single spatial frequency in the detector plane. The fringe observables are extracted from the Fourier transform of the interferogram (see Fig. 2). Each observable is a fringe complex visibility. The preservation of the nonredundancy relation ensures that the extracted fringes can be used to form closure phases (Jennison 1958; Cornwell 1989). The compelling advantage of the use of closure phase data is the rejection of any residual pupilplane phase errors, which are the source of both AO PSF cal- 


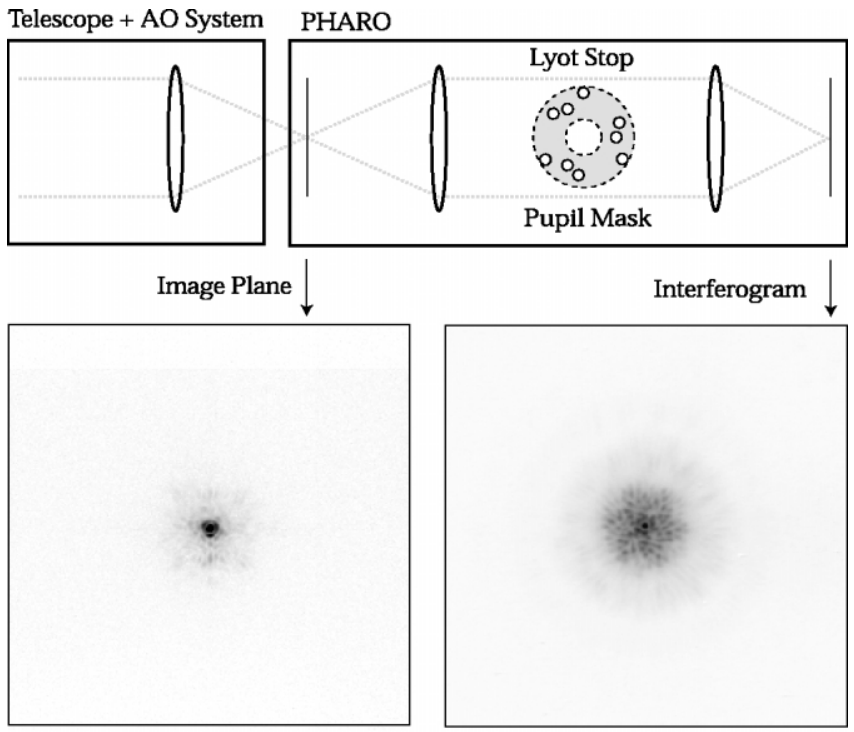

FIG. 1.-Functional diagram of the aperture masking experiment. Although in practice the optical system is complex and involves many reflective optical elements, only the essential imaging properties are represented with lenses. After the telescope and AO system, the telescope pupil is reimaged at the PHARO Lyot stop. Under most common circumstances, the Lyot stop serves only as a cold baffle. In a coronagraphy mode, an undersized stop is used to block the light diffracted by the pupil edges. For the aperture masking interferometry mode, a pupil mask is placed in the Lyot stop to form an interferogram recorded at the focal plane. The images shown are the full pupil AO images and nine-hole interferograms obtained for GJ 802 in 2004 September. [See the electronic edition of the Journal for a color version of this figure.]

ibration difficulties and speckle noise. The nonredundant masking technique therefore rejects the phase noise associated with both the instantaneous and time-averaged AO system performance. Images can be reconstructed using self-calibration techniques. Although the dynamic range achieved here is modest by comparison with conventional $\mathrm{AO}$, it is uniquely close to the central star, within a few $\lambda / D$, which is an area not accessible to coronagraphs. The use of closure relations in radio interferometry has enabled imaging with dynamic range exceeding $10^{5}$ (e.g., Hardcastle et al. 2003).

\subsection{GJ 802 Observations}

GJ 802 was observed at the Palomar 200" telescope with conventional AO imaging and nine-hole aperture masking interferometry on 2004 September 2 UT, in good seeing. Uncompensated images earlier in the night showed 0"6 FWHM seeing at $H$ band ( 0.75 in $V$ band). The conventional AO imaging placed an upper limit of a contrast ratio of 0.05 for any companion at $\sim 100$ mas (Pravdo et al. 2005). Imaging observations have also been attempted with Keck laser guide star AO system (C. Gelino 2006, private communication) and the Hubble Space Telescope (GO-10517), but they have not detected the companion.

Interferograms were recorded using the Fowler sampling mode of PHARO on a $256 \times 256$ subarray. PHARO provides a mode whereby all reads of a Fowler sampling sequence can be saved. We use this mode and the minimum exposure time to save a data cube of 16 sequential nondestructive reads of the detector without reset. This provides 15 pairwise, $431 \mathrm{~ms}$ exposures in a total exposure time of $6465 \mathrm{~ms}$, thus very efficiently recording a large number of short exposures, so long as the detector does not saturate in the total exposure time. For

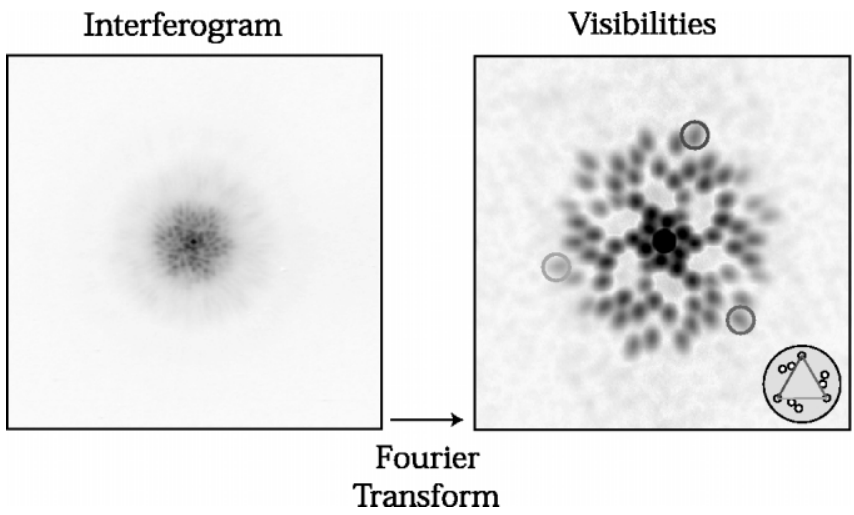

FIG. 2.-Extraction of closure phases from optical interferograms. Each "splodge" in the Fourier plane corresponds to the fringe formed by a pair of holes in the pupil mask. The splodges are point-symmetric since the interferogram is real (or equivalently, each splodge appears for both the baseline formed by a pair of holes in both order A-B and B-A). Since the splodges map uniquely to a baseline, closure phase relations can be constructed that reject any residual phase errors and therefore speckle noise. One such closure phase triangle (out of 84 possible for a nine-hole mask) is shown in the lower right with the visibility splodges corresponding to each baseline circled. [See the electronic edition of the Journal for a color version of this figure.]

these observations, we recorded 115 image cubes yielding $1725,431 \mathrm{~ms}$ exposures on source. The large number of frames allows good estimation of the errors. The observations show $0.6 \mathrm{rms}$ closure phase scatter.

Calibration of the interferograms is achieved by observing an unresolved source to measure the system visibilities. It is usually considered necessary to choose a calibrator that is as similar as possible to the target star in both wave front sensor (approximately $R$ band) and science camera ( $H$ band) brightness, at similar air mass. We select calibrator stars by searching the USNO CCD Astrograph Catalog (UCAC2; Zacharias et al. 2004) and Two Micron All Sky Survey (2MASS) catalog for stars nearby in the sky with similar properties. The UCAC2 catalog bandpass is between the $V$ and $R$ bands and for practical purposes has proven to be a similar magnitude scale to the PALAO wave front sensor. For these observations 2MASS 20494024+4526398 (2UCAC 47204238; UC = $14.72 \mathrm{mag}$; $H=7.73 \mathrm{mag}$ ) was selected as a calibrator star.

\subsection{Data Analysis}

The data were dark-subtracted, flat-fielded, and analyzed with a custom software pipeline written in IDL. The pipeline outputs a bispectrum in OIFITS format (Pauls et al. 2005). A binary model is fit to the bispectrum with a reduced $\chi^{2}$ method. In practice, we have found that the visibility amplitude calibration is poor, and superior results are achieved with a fit to the closure phase alone. Presumably this is because the visibility amplitude calibration is susceptible to the same fluctuations in seeing and $\mathrm{AO}$ performance between source and calibrator that plague conventional imaging with AO. As discussed in $\S 2.1$, the closure phase rejects the residual phase errors and is therefore expected to be robust. Although in principle the closure phase is self-calibrating, there are systematic nonzero closure phase errors of a few degrees. Therefore, it remains necessary to calibrate the nonzero closure phases. The source of these nonzero closure phases is not entirely understood, but the level is consistent with the expected telescope and $\mathrm{AO}$ system residual wave front errors and detector flat-fielding errors. Once the visibility amplitude is rejected from the analysis, it is also possible to include additional calibrators observed 


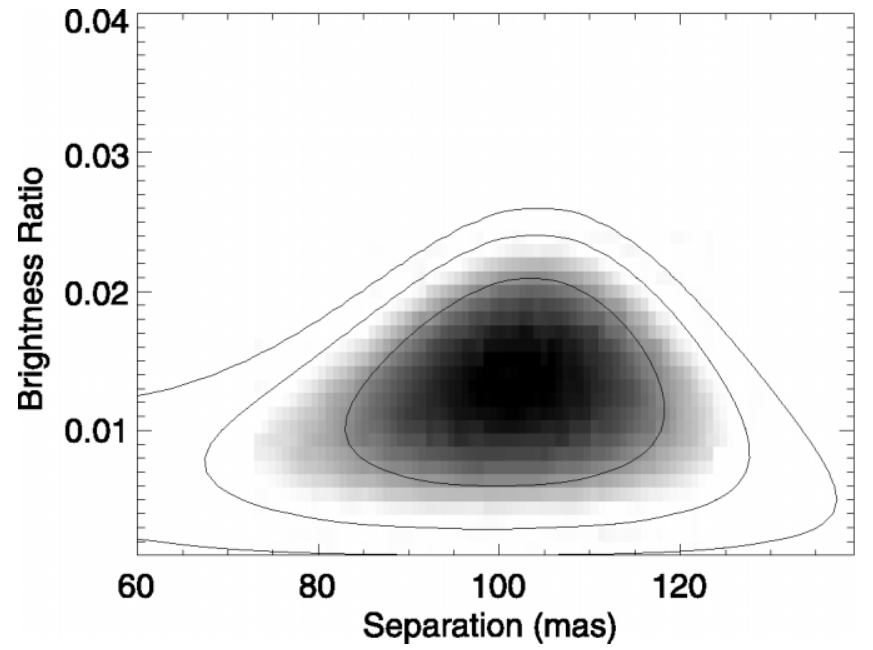

FIG. 3.-Likelihood function cross section for GJ 802 binary model fit. The contours represent $90 \%, 99 \%$, and $99.9 \%$ confidence levels.

throughout the night. These additional calibrators usually improve the estimation of errors and provide a robustness against possibility that the calibrator itself is an unknown binary. We include observations of HD 4915 (G0 V, $V=6.76, H=5.416) \mathrm{HD} 28005$ (G0 V, $V=6.71, H=5.506)$ as additional calibrators in this analysis.

Likelihood contours for the binary model parameters are shown in Figure 3. The derived binary model is separation $102 \pm 7$ mas at position angle $36.1 \pm 4.5$. The $H$-band contrast ratio is $74.4+/-18.5(\Delta H=4.68 \pm 0.28 \mathrm{mag})$.

\section{ORBITAL PARAMETERS}

The measured position of GJ 802B, shown in Figure 4, is very near the expected position from Pravdo et al. (2005), although this prior information is not involved in the fit to the closure phases used to extract the astrometry. Updated orbital elements combining this resolved observation and additional astrometric observations since Pravdo et al. (2005) are shown in Table 1. This orbit is consistent with the pure photocenter astrometry orbit derived in Pravdo et al. (2005).

\section{DISCUSSION}

The luminosity of GJ $802 \mathrm{~B}$ can be determined precisely by a differential measurement from GJ 802A. The 2MASS catalog (Cutri et al. 2003) records the $H$-band brightness of GJ 802 as $9.058 \pm 0.019$ mag. Adopting the parallax determined by Pravdo et al. (2005) of $64 \pm 2$ mas, the absolute magnitude of GJ 802 is $M_{H}=8.11 \pm 0.07 \mathrm{mag}$. Using the $H$-band contrast ratio of $\Delta H=4.68 \pm 0.28 \mathrm{mag}$, we determine the absolute magnitude of GJ $802 \mathrm{~B}$ to be $M_{H}=12.79 \pm 0.3$. Comparison with models of Baraffe et al. (2003) admits a large range of possible masses depending on age (see Fig. 5), 0.057-0.074 $M_{\odot}$, with $T_{\text {eff }}=$ $1850 \pm 50 \mathrm{~K}$ for models of ages $1-10 \mathrm{Gyr}$. With an age estimate of $<6$ Gyr based on activity (Pravdo et al. 2005), the Baraffe models indicate a mass $\sim 0.07 M_{\odot}$.

For models with age $>5 \mathrm{Gyr}$, the mass range consistent with this luminosity is remarkably narrow, 0.072-0.074. This modeldependent mass range is narrower than the present dynamical mass determinations based on the STEPS orbit alone (Pravdo et al. 2005) or this work. Although this mass is consistent with the orbital solution based on the STEPS and AO masking result,

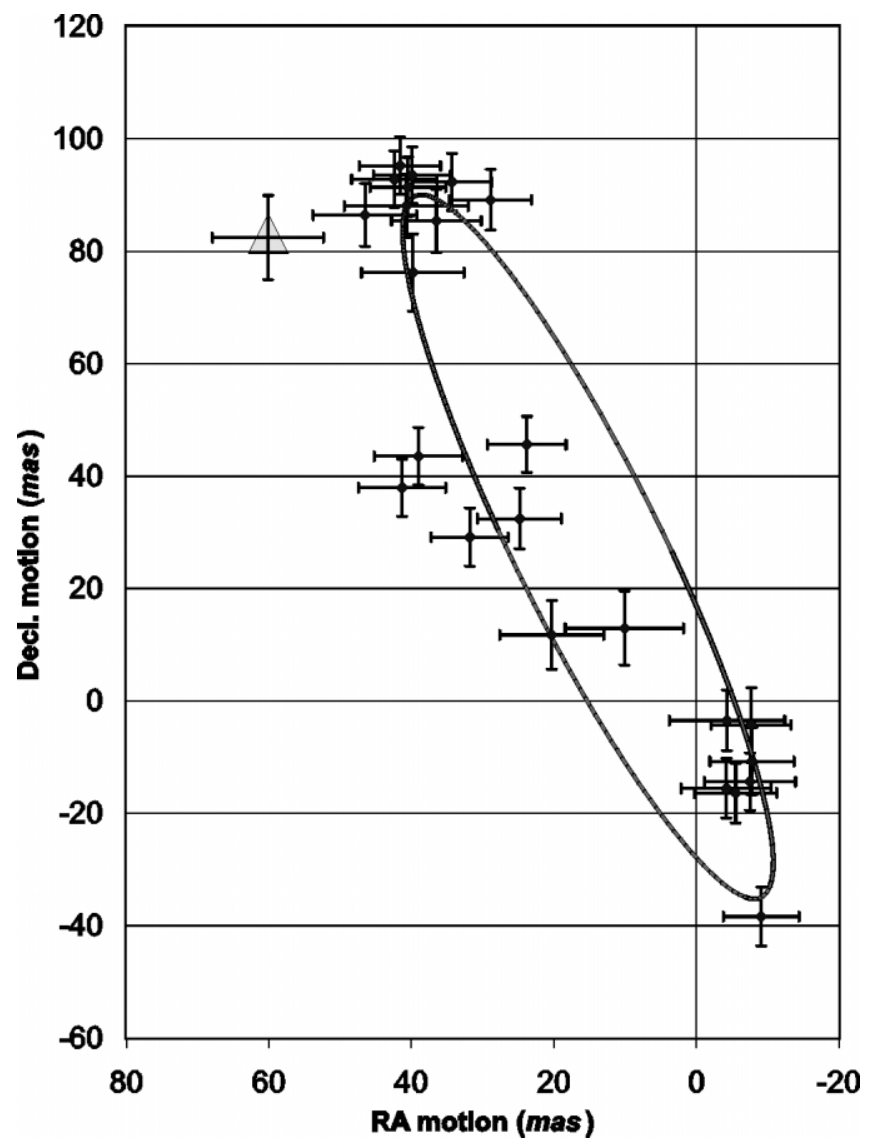

FIG. 4.-Orbit of GJ 802B. STEPS astrometric observations are diamonds. The uncertainties shown are the photocenter astrometry uncertainties multiplied by the ratio $(\sim 4)$ of the displayed Keplerian orbit (curve) to the photometric orbit. The resolved AO observation is the triangle. [See the electronic edition of the Journal for a color version of this figure.]

the high mass required by the models if GJ 802 is old demands an unusually high eccentricity $(e>0.8)$ for the astrometric orbital solution. It is therefore tempting to conclude that the GJ 802 is young $(<1 \mathrm{Gyr})$, which would admit a lower eccentricity for the orbital solution.

A sample of field objects selected with a luminosity near the substellar limit would be dominated by stellar objects since the cooling time of low-mass stars dramatically exceeds that of brown dwarfs. Further, since the cooling time increases with mass, the distribution of field objects below the substellar limit contains many more old massive brown dwarfs than young

TABLE 1

Orbital ElEmEnts

\begin{tabular}{|c|c|}
\hline Quantity & Value \\
\hline Absolute parallax (mas) & $64.5 \pm 2$ \\
\hline Proper motion (mas) ... & $1933 \pm 1$ \\
\hline Position angle (deg) . & $26.6 \pm 0.5$ \\
\hline Period $(\mathrm{yr}) \ldots \ldots \ldots$ & $3.13 \pm 0.04$ \\
\hline Total mass $\left(M_{\odot}\right) \ldots$ & $0.24 \pm 0.05$ \\
\hline Semimajor axis (AU) & $1.32 \pm 0.09$ \\
\hline Eccentricity .......... & $0.60 \pm 0.29$ \\
\hline Inclination $(\mathrm{deg}) \ldots \ldots \ldots \ldots \ldots \ldots \ldots \ldots \ldots \ldots$ & $82 \pm 2$ \\
\hline Longitude of ascension node (deg) ....... & $18.5 \pm 4.5$ \\
\hline Argument of periastron $(\mathrm{deg}) \ldots \ldots \ldots \ldots$ & $222.5 \pm 25.5$ \\
\hline 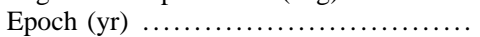 & $2000.51 \pm 0.19$ \\
\hline Primary mass $\left(M_{\odot}\right) \ldots \ldots$ & $0.175 \pm 0.021$ \\
\hline Secondary mass $\left(M_{\odot}\right) \ldots \ldots \ldots \ldots \ldots \ldots$ & $0.064 \pm 0.032$ \\
\hline
\end{tabular}




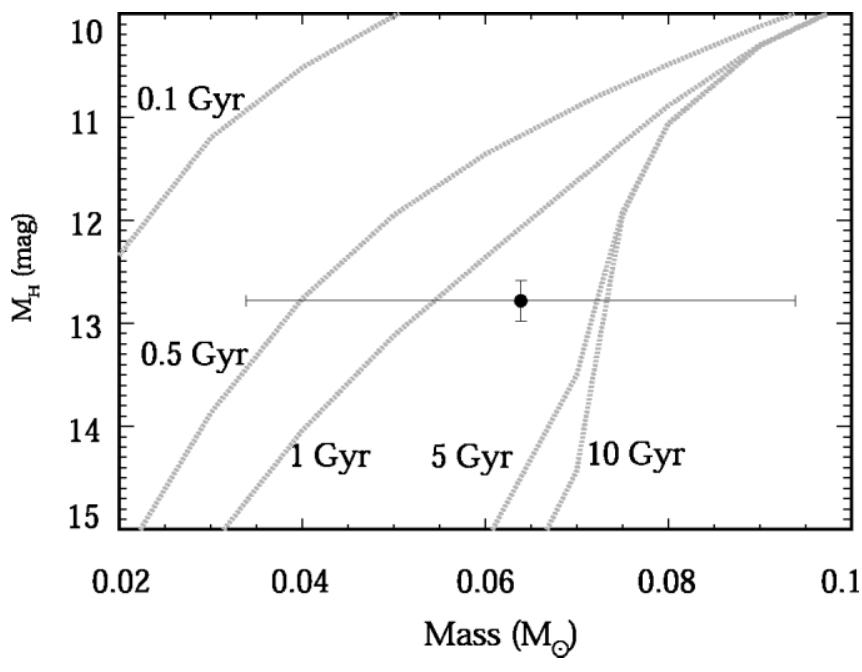

FIG. 5.-Observed mass and luminosity of GJ 802B compared to theoretical isochrones from Baraffe et al. (2003). The mass is the dynamical mass on the basis of the orbital fit shown in Table 1 and Fig. 4. Uncertainty in the mass is largely due to the poorly constrained orbital eccentricity. Future observations should constrain the eccentricity and therefore the mass precisely.

low-mass objects. The conclusion that GJ 802 is young would be remarkably puzzling since then the only three resolved binaries with dynamical masses below $0.08 M_{\odot}$ (GJ 802B, GL 569 Bab [Zapatero Osorio et al. 2004; Simon et al. 2006], and 2MASSW J0746425+2000321 AB [Bouy et al. 2004]) are in- ferred to be young brown dwarfs, despite the fact that young low-mass objects are less likely to be found than old highmass ones. These conclusions are suggestive that the models are underpredicting the luminosity of substellar objects.

Ultimately, further observations will provide model-constraining dynamical measurements of the masses of both the primary and secondary. If GJ 802 is an old star, then GJ 802B is remarkably close to the brown dwarf/substellar boundary, and more measurements with this technique will provide tight constraints on the substellar evolutionary models.

We thank Rick Burruss, Jeff Hickey, and the Palomar observatory staff for help with these observations. This work is partially funded by the National Science Foundation under grants AST 03-35695 and AST 05-06588. The research described in this Letter was performed in part by the Jet Propulsion Laboratory, California Institute of Technology, under contract with the National Aeronautics and Space Administration. This publication makes use of data products from the Two Micron All Sky Survey, which is a joint project of the University of Massachusetts and the Infrared Processing and Analysis Center/California Institute of Technology, funded by the National Aeronautics and Space Administration and the National Science Foundation.

\section{REFERENCES}

Baraffe, I., Chabrier, G., Barman, T. S., Allard, F., \& Hauschildt, P. H. 2003, A\&A, 402, 701

Bouy, H., et al. 2004, A\&A, 423, 341

Brandner, W., Apai, D., Lenzen, R., Feldt, M., \& Huélamo, N. 2005, in ASP Conf. Ser. 343, Astronomical Polarimetry: Current Status and Future Directions, ed. A. Adamson, C. Aspin, \& C. Davis (San Francisco: ASP), 75

Burgasser, A. J., Reid, I. N., Siegler, N., Close, L., Allen, P., Lowrance, P., \& Gizis, J. 2006, preprint (astro-ph/0602122)

Carson, J. C., Eikenberry, S. S., Brandl, B. R., Wilson, J. C., \& Hayward, T. L. 2005, AJ, 130, 1212

Close, L. M., et al. 2005, Nature, 433, 286

Cornwell, T. J. 1989, Science, 245, 263

Cutri, R. M., et al. 2003, 2MASS All Sky Catalog of Point Sources (Pasadena: IPAC/Caltech), http://irsa.ipac.caltech.edu/applications/Gator/

Fitzgerald, M. P., \& Graham, J. R. 2006, ApJ, 637, 541

Hardcastle, M. J., Worrall, D. M., Kraft, R. P., Forman, W. R., Jones, C., \& Murray, S. S. 2003, ApJ, 593, 169

Hayward, T. L., Brandl, B., Pirger, B., Blacken, C., Gull, G. E., Schoenwald, J., \& Houck, J. R. 2001, PASP, 113, 105

Jennison, R. C. 1958, MNRAS, 118, 276

Lloyd, J. P., et al. 2001, Proc. SPIE, 4490, 290

Macintosh, B. A., Becklin, E. E., Kaisler, D., Konopacky, Q., \& Zuckerman, B. 2003, ApJ, 594, 538

Macintosh, B. A., et al. 2004, Proc. SPIE, 5490, 359

Marois, C., Doyon, R., Racine, R., \& Nadeau, D. 2000, PASP, 112, 91

Marois, C., Lafrenière, D., Doyon, R., Macintosh, B., \& Nadeau, D. 2006, ApJ, 641, 556

Marois, C., Racine, R., Doyon, R., Lafrenière, D., \& Nadeau, D. 2004, ApJ, 615, L61
Nakajima, T., Kulkarni, S. R., Gorham, P. W., Ghez, A. M., Neugebauer, G., Oke, J. B., Prince, T. A., \& Readhead, A. C. S. 1989, AJ, 97, 1510

Oppenheimer, B. R., Dekany, R. G., Hayward, T. L., Brandl, B., Troy, M., \& Bloemhof, E. E. 2000, Proc. SPIE, 4007, 899

Pauls, T. A., Young, J. S., Cotton, W. D., \& Monnier, J. D. 2005, PASP, 117, 1255

Pravdo, S. H., \& Shaklan, S. B. 1996, ApJ, 465, 264

Pravdo, S. H., Shaklan, S. B., \& Lloyd, J. 2005, ApJ, 630, 528

Racine, R., Walker, G. A. H., Nadeau, D., Doyon, R., \& Marois, C. 1999, PASP, 111,587

Readhead, A. C. S., Nakajima, T. S., Pearson, T. J., Neugebauer, G., Oke, J. B., \& Sargent, W. L. W. 1988, AJ, 95, 1278

Simon, M., Bender, C., \& Prato, L. 2006, ApJ, 644, 1183

Sivaramakrishnan, A., Koresko, C. D., Makidon, R. B., Berkefeld, T., \& Kuchner, M. J. 2001, ApJ, 552, 397

Soummer, R., et al. 2006, in IAU Colloq. 200, Direct Imaging of Exoplanets: Science and Techniques, ed. C. Aime \& F. Vakili (Cambridge: Cambridge Univ. Press), 581

Sparks, W. B., \& Ford, H. C. 2002, ApJ, 578, 543

Troy, M., et al. 2000, Proc. SPIE, 4007, 31

Tuthill, P. G., Lloyd, J. P., Ireland, M. J., Martinache, F., Monnier, J. H. W., ten Brummelaar, T., Turner, N., \& Townes, C. 2006, Proc. SPIE, 6272, 103

Tuthill, P. G., Monnier, J. D., Danchi, W. C., Wishnow, E. H., \& Haniff, C. A. 2000, PASP, 112, 555

Zacharias, N., Urban, S. E., Zacharias, M. I., Wycoff, G. L., Hall, D. M., Monet, D. G., \& Rafferty, T. J. 2004, AJ, 127, 3043

Zapatero Osorio, M. R., Lane, B. F., Pavlenko, Y., Martín, E. L., Britton, M., \& Kulkarni, S. R. 2004, ApJ, 615, 958 